Wiener Klinisches Magazin 2014 · 17:3

DOI 10.1007/s00740-014-0225-9

C) Springer-Verlag Wien 2014

\section{Kienast}

Springer-Verlag, Wien

\title{
Das "Geschäft" mit der Angst
}

Es gibt zu viele Ärzte - es gibt zu wenige Ärzte, zu viele Lehrer - zu wenige Lehrer. Spezialisierung ist gefragt. Generalisten braucht das Land. So wird Nachwuchs nachhaltig verunsichert. Das „Geschäft" mit der Angst funktioniert noch immer und erstaunlicherweise scheinen sich Entscheidungsträger lieber auf die Kraft der negativen Emotionen zu verlassen, denn auf jene der positiven. Gute Aussichten haben, zumindest in unseren Breiten, offenbar weniger Überzeugungskraft als die Bedrohung - als Auslöser das Gegenteil zu tun.

\section{Wie das Monster von Loch Ness}

Das seit Jahren bewusste, immer wieder kritisierte und dennoch weiter bestehende Problem der viel zu langen Arbeitszeiten von Spitalsärzten ist durch eine Rüge auf EU-Ebene wieder einmal virulent geworden. Übermüdete Ärzte, die fehleranfälliger sind als ihre ausgeruhten Kollegen, weil sie Wochenarbeitszeiten von 80 und mehr Stunden absolvieren, tauchen in der medialen Berichterstattung mit einer Regelmäßigkeit auf, die an das Ungeheuer von Loch Ness erinnern. Bisweilen brüsten sich Marathon-Dienst-Absolventen mit der Länge ihrer ununterbrochenen Arbeitszeit und überbieten sich gegenseitig, viele sind schlichtweg erschöpft.

Die Spitalserhalter geben zu bedenken, dass eine Einhaltung der Höchstarbeitszeit von 48 Wochenstunden einen enormen zusätzlichen Personalbedarf bewirken würde - für dessen Deckung der Nachwuchs fehlt und der überdies finanziell problematisch werden könnte. Schon spricht man von einem Turnusärztemangel. Eben waren es noch lange Wartelisten und ein Punkte- system, um einen der begehrten Turnusarztplätze vor allem im Raum Wien zu bekommen. Gleichzeitig wird die Anerkennung von Schwerpunktspitälern als Ausbildungskrankenhäuser durch bürokratische Hindernisse erschwert. Ziemlich viele Widersprüche....

\section{Der Wunsch nach Arbeitszufriedenheit wächst}

Aber die Erwartungen junger Menschen an ihr Arbeits- und Privatleben haben sich in den vergangenen Jahrzehnten ebenfalls verändert, und das betrifft sehr stark auch jene, die eine universitäre Ausbildung anstreben, absolvieren oder bereits beendet haben. Arbeit soll nicht nur eine Einkommensquelle sein, sondern auch Zufriedenheit geben und sie soll das Privatleben nicht ersticken. Arbeit und Privatleben verschränken sich zwar durch beinahe permanente Verfügbarkeit mittels moderner Medien, das Bewusstsein für eine Vereinbarkeit von Beruf und Privatleben ist aber stärker geworden. Es gibt ein Leben jenseits der durchaus erfüllenden Arbeit - Familie, Hobbys, Kultur, Sport, soziales Engagement. Pausen sind absolut notwendig. Die Realität sieht anders aus. Vom Berufseinsatz völlig erschöpfte Menschen haben für derartige Aktivitäten keine Kapazitäten mehr - oft holen sich Körper und Geist die nötige Ruhe im Nervenzusammenbruch.

\section{Auf zu hohe Ansprüche folgt der Zusammenbruch}

Gerade in helfenden Berufen ist die Rate der „Burn-out“-Fälle bekanntlich überproportional hoch im Vergleich zur Ge- samtbevölkerung. Auch weil die eigenen Ansprüche extrem hoch und daher dauerhaft schwer erfüllbar sind. Dann wird Arbeit und alles, was damit zusammen hängt, zur Last.

Berufsentscheidungen sind heute für viele junge Menschen von rationalen Überlegungen geleitet: Wie die Chancen am Arbeitsmarkt sind, welche Tradition in der Familie gepflegt wird, welche Einkommenserwartungen bestehen und letztlich auch in vielen Fällen Prestigegründe. Ob die Arbeitszufriedenheit durch solche äußeren Rahmenbedingungen gefördert wird, sei dahin gestellt. Die immaterielle Begeisterung und eine glückliche Vision sollten jedenfalls wieder mehr Bedeutung gewinnen

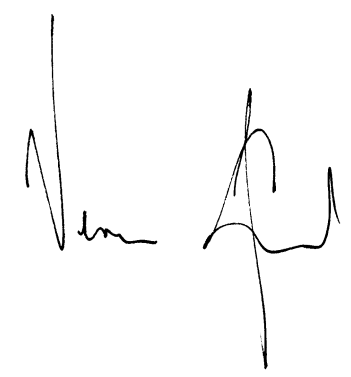

meint Ihre

Verena Kienast

Springer-Verlag GmbH

SpringerMedizin/Editor

verena.kienast@springer.at 